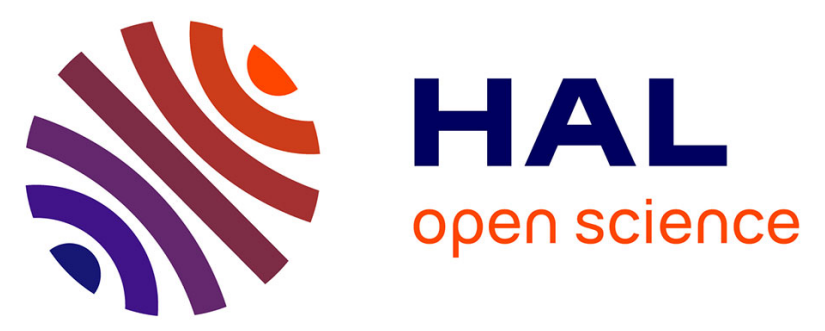

\title{
A new adaptive secondary mirror for astronomy on the University of Hawaii 2.2-meter telescope
}

Mark R Chun, Christoph Baranec, Olivier Lai, Jessica R Lu, Ruihan Zhang, Stefan Kuiper, Wouter Jonker, Matthew Maniscalco

\section{- To cite this version:}

Mark R Chun, Christoph Baranec, Olivier Lai, Jessica R Lu, Ruihan Zhang, et al.. A new adaptive secondary mirror for astronomy on the University of Hawaii 2.2-meter telescope. SPIE Astronomical Telescopes + Instrumentation, Dec 2020, Online Only, France. pp.52, 10.1117/12.2562847 . hal03371091

\section{HAL Id: hal-03371091 \\ https://hal.science/hal-03371091}

Submitted on 28 Oct 2021

HAL is a multi-disciplinary open access archive for the deposit and dissemination of scientific research documents, whether they are published or not. The documents may come from teaching and research institutions in France or abroad, or from public or private research centers.
L'archive ouverte pluridisciplinaire HAL, est destinée au dépôt et à la diffusion de documents scientifiques de niveau recherche, publiés ou non, émanant des établissements d'enseignement et de recherche français ou étrangers, des laboratoires publics ou privés. 


\title{
A new adaptive secondary mirror for astronomy on the University of Hawaii 2.2-meter Telescope \\ Mark Chun*a, Christopher Baranec ${ }^{\mathrm{a}}$, Olivier Lai ${ }^{\mathrm{b}}$, Jessica R. Luc ${ }^{\mathrm{c}}$, Ruihan Zhang ${ }^{\mathrm{a}}$, Stefan Kuiper ${ }^{\mathrm{d}}$, Wouter Jonkerd, Matthew Maniscalco $^{\mathrm{d}}$ \\ ${ }^{a}$ University of Hawaii at Manoa, 640 N. Aohoku Place, Hilo, HI, USA 96720; ' Observatoire de la \\ Cote d'Azur, France; 'University of California, Berkeley, USA, 'TNO, Nederlands
}

\begin{abstract}
We are developing a new adaptive secondary mirror (ASM) for the University of Hawaii 2.2-meter telescope based on a novel and very efficient hybrid variable reluctance actuator developed by TNO. The actuator technology has broad implications on the ASM design and results in an ASM with a thicker facesheet, lower power dissipation, and simple controls. We report here preparations and plans for lab testing as well as on-sky demonstration of the ASM. The lab calibrations of the ASM influence functions will use a phase measuring deflectometry setup. The on-sky tests will include the evaluation of the use of the ASM for narrow field AO observations at visible through near infrared wavelengths, for very wide fields of view ground-layer adaptive optics, and for seeing limited non-adaptive optics observations.
\end{abstract}

Keywords: adaptive secondary mirror

\section{INTRODUCTION}

Adaptive secondary mirrors (ASM) greatly enhance two key observational capabilities for ground-based adaptive optic systems: low-thermal emissivity background observations and wide-field of view seeing-improved observations. Telescopes currently with ASMs include the MMT ${ }^{1}, \mathrm{LBT}^{2}, \mathrm{Magellan}^{3}$, and recently VLT ${ }^{4}$. The current ASMs have relatively large actuator densities so high levels of wavefront correction can be obtained. The principal downside of the current ASMs is that their use of a voice-coil actuator adds complexity to the systems making it difficult for them to meet the very stringent reliability requirements demanded of the telescope optics.

Here, we present the status and plans for testing the new adaptive secondary mirror we're building for the University of Hawaii 2.2-meter telescope (UH88). The ASM project is an on-sky demonstrator for an actuator technology developed by TNO and that we believe is an ideal match to adaptive secondaries. The goal of the project is to demonstrate the technology's suitability for astronomical adaptive optics in various AO modes (single-conjugate adaptive optics and wide-field ground-layer adaptive optics), and as a fixed secondary for seeing limited observations. The project is a collaboration between TNO, the University of Hawaii's Institute for Astronomy, and our industrial partners VDL and Hyperion.

The project is centered about TNO's hybrid variable reluctance actuators ${ }^{5}$. The key features of these actuators are that they produce a large force output for a given current making them capable of producing large strokes in very efficient packaging volumes. TNO has demonstrated the actuators in a $160 \mathrm{~mm}$-diameter deformable mirror with an $18 \mathrm{~mm}$ actuator pitch. Within the linear regime of these actuators $(+/-150 \mathrm{~mA})$, the force output of the actuators is about $+/-8 \mathrm{~N}$. This is about an order of magnitude more force typical of voice-coil actuators used in current adaptive secondaries.

The efficiency and output force of these actuators make them well suited to ASMs and lead to important improvements in the optical facesheet and the controls of the ASM compared to current adaptive secondaries. The optical facesheets in current ASM are thin $(\sim 1.6 \mathrm{~mm})$ due to the lower force output of voice-coil actuators. This makes them fragile to handle and difficult to fabricate. The higher force output of the TNO actuators allow the optical face sheet to be thicker. In the case of the UH88 ASM the facesheet will be $\sim 3.5 \mathrm{~mm}$ thick, similar to a car windshield thickness, and still provide many tens of microns of stroke. This makes for an optical facesheet that is easier and safer to handle. The large stroke provided

*markchun@hawaii.edu; phone 1808 932-2317; 
by the actuators also eases the fabrication requirements on the optical facesheet. Rather than grind a large piece of glass down to the final thickness and shape, the facesheet for the UH88 ASM will be slumped on a master mold and MHD polished to remove high-frequency residual errors. We believe this process has a significant cost savings and is scalable to large aperture ASMs. The higher force output is expected to lead to a simpler control system. The TNO actuator+facesheet combination in the UH88 ASM design is mechanically stiff due to the thickness of the facesheet, the inherent stiffness of the actuators, and the fact that the actuators are mechanically connected to the facesheet. This is in contrast with current adaptive secondaries where the thin facesheet magnetically floats over the reference body and the mechanical stiffness is achieved by adding an additional capacitive position sensors/servos that control the position of the actuator+facesheet. This position servo loop is needed to translate the lower force output of the voice coil actuators into a position displacement but produces a system that goes-to and maintains a commanded shape. This makes it effectively a stiff system. The cost in doing this is added system complexity with the sensors, servo electronics, thin gap between the facesheet and optical reference body, and a considerable heat load at the top end of the telescope due to the servo electronics. The TNO actuator/ASM avoids the use of this extra control system and its complexity and power dissipation. For comparison, the ASM and drive electronics for the UH88 ASM will dissipate about 20-30W. This is more than an order of magnitude less than that for a similarly sized ASM based on voice coil actuators. In addition most of this dissipation is in the drive electronics which does not need to be colocated with the ASM. Finally, the greater force output of the TNO actuators provides flexibility in the design of the ASM. In particular the force output is large enough that the spacing of the actuators can be set by the requirements of the desired adaptive optics correction rather than the need to be spaced to mechanically support a thin optical facesheet from deformations due to gravity.

\section{BASIC REQUIREMENTS OF THE UH88 ASM}

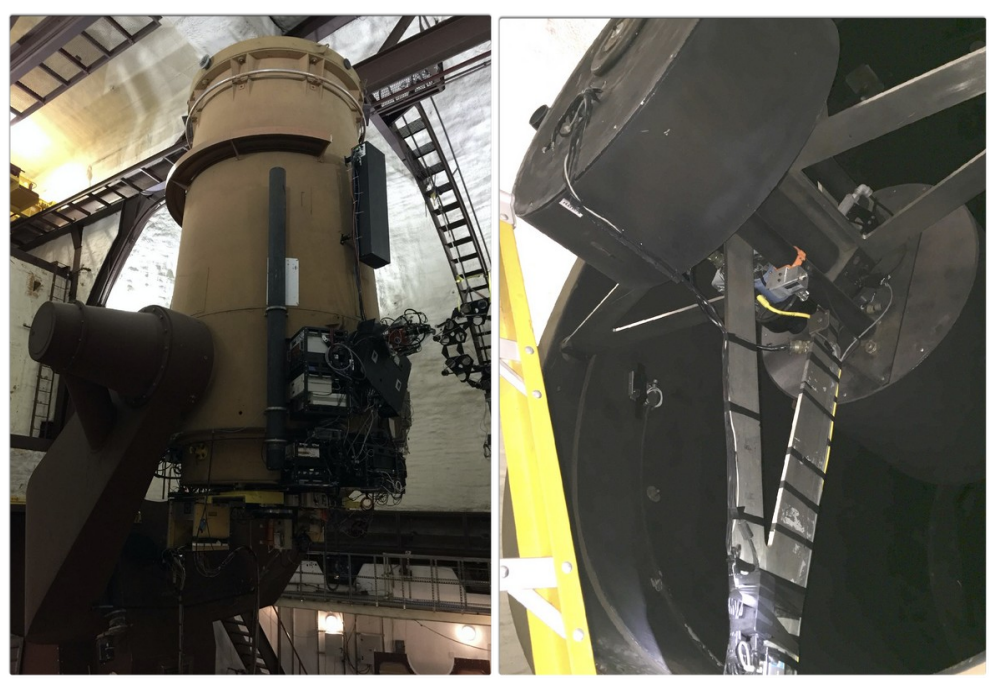

Figure 1. (LEFT) The University of Hawaii 2.2-meter telescope (LEFT) shown with the Robo-AO system mounted to the North bent-Cassegrain port. The Robo-AO Rayleigh laser is the long black rectangular box seen midway up the side of the telescope tube. (RIGHT) The top-end of the telescope showing its two secondary mirrors. The secondary currently "in the beam" is the f/ 10 fixed secondary. The secondary out of the beam (pointed away from the telescope tube) is the no longer used Coude secondary. The UH88 ASM will replace this Coude secondary. The top-end can rotate ( $30 \mathrm{~min})$ to place either secondary in the beam.

The goal of the UH88 ASM project is to build an ASM around the TNO technology and demonstrate its performance and operational readiness in a number of AO modes and for seeing limited observations. The ASM will be deployed on the University of Hawaii 2.2-meter telescope (Figure 1). While this telescope just celebrated its 50th year of operation, it has a number of features that make this a nice match for the development of an ASM. The telescope is on Mauna Kea, Hawaii next to 4-meter and 8-/10-meter class telescopes. The UH 2.2-meter telescope is operated by the Institute for Astronomy and we can dedicate, and historically have dedicated, on-sky time to astronomical technology development. 
The telescope is home to two adaptive optics systems: Robo- $\mathrm{AO}^{6}$ and soon Robo-AO-27, a Rayleigh laser guide star AO system with both visible and near-infrared science cameras, and 'imaka ${ }^{8}$, a ground-layer testbed/demonstrator with 3-5 NGSs and a visible-light CCD imager covering $11 \times 11$ arcminute FOV in the center. In addition the telescope has two seeing limited instruments - an integral field visible light spectrograph and a wide-field imager $\left(0.25^{\circ} \times 0.25^{\circ}\right)$. The telescope's equatorial mount simplifies the ASM control since the orientation of the ASM is fixed in the WFS(s).

In order to feed all of these instruments the ASM will replicate the currently used f/10 secondary. The resulting ASM is relatively large for a telescope of this size $(620 \mathrm{~mm}$ in diameter) but this provides a large field of view at the Cassegrain focal plane that allows us to test very wide field GLAO with an unvignetted field of view of 0.5 degrees.

The ASM will interface with the existing secondary interface plate. The telescope has two secondaries mounted at all times and the top end can "flip" to put either into the beam (Figure 1). For most of the first 50 years, the two secondaries were (1) for the Cassegrain focus (f/10) and (2) for the Coude feed. The Coude feed is no longer used so it will be replaced by an ASM that optically replicates the $\mathrm{f} / 10$ secondary. We can then "flip" between the fixed and adaptive secondaries in approximately 30 minutes. This gives us flexibility in when/how we use the ASM. It also keeps the ASM continuously exposed to the observatory environment allowing us to test the long-term operability and reliability in such conditions. While this approach has obvious advantages, it does have an important design constraint. The volume, location, and mass of the ASM must be similar to the replaced secondary to maintain the balance of the top end and to put the secondary within the range of the existing focusing mechanism. The volume requirement has an important impact on the dynamic behavior of the ASM that we will discuss in the next section.

The AO specifications of the ASM are driven by the desire to integrate it with the Robo-AO-2 wavefront sensors (WFS) and control system and to provide correction at visible and near-infrared wavelengths. The ASM's 210 actuators in a circular arrangement is well matched to the $16 \times 16$ subaperture Shack-Hartmann WFSs in the RoboAO-2 system and the dynamic response of the ASM will be consistent with a 0-dB closed loop bandwidth of about $100 \mathrm{~Hz}$. This should give good performance into the red end of the visible on a 2.2-meter telescope. The stroke requirements for the ASM are driven more by the desire to have sufficient range to accommodate any residual loworder aberrations in the fabrication of its slumped facesheet. Nominally we specified this to be $+/-15$ microns and $+/-4$ microns inter-actuator stroke.

\section{DESIGN AND STATUS}

Several other contributions in these proceedings cover the design and current fabrication status ${ }^{9}$, and performance estimates $^{10}$. Here we highlight a few of items as they apply to the suite of tests we plan for the system. For more details we refer you to Jonker et al. ${ }^{9}$ and Kuiper et al. ${ }^{10}$

The key high-level design features of the UH88 ASM are:

- A slumped facesheet $3.5 \mathrm{~mm}$ thick

- Struts that connect the face sheet to the actuators

- An aluminum-alloy support structure that holds the actuators and provides the mechanical base.

- 210 actuators in a radial arrangement with a $39 \mathrm{~mm}$ pitch

- An update rate of $2 \mathrm{kHz}$ and a full assembly response consistent with an $\mathrm{AO}$ system $0 \mathrm{~dB}$ bandwidth of $100 \mathrm{~Hz}$.

- An interface to the existing telescope interface plate.

TNO has modeled the performance of the system ${ }^{10}$. Some key aspects of the modeling include a detailed analysis of the surface figure errors due to changes in the telescope orientation, changes in temperature, epoxy print-thru, and the actuator stroke/stiffness. TNO has done breadboarding/prototyping has been done to ground the results ${ }^{9}$. The dynamic behavior of the system has been modeled both for the ASM and for the telescope top-end. The lowest resonant mode of the system is due to flexure of the ASM structure/body and is a tip/tilt mode at about $160 \mathrm{~Hz}$. This is a relatively low 
frequency but (1) its well above the bandwidth we need for tip/tilt correction on the 88" telescope and (2) we believe that we can suppress the resonance of this particular mode with relatively simple control algorithms. In particular we plan to control the tip/tilt modes with a separate control gain than the higher order modes ${ }^{10}$. We note that this resonant frequency is largely set by the design of the ASM support structure and this is constrained by the requirement that the UH88 ASM fit within the existing volume of the fixed secondary it replaces. Relaxing this requirement and making the ASM structure/body thicker or from a stiffer material would move this resonance to higher frequencies so the low resonance seen in the UH88 ASM is not fundamental to the approach. The first resonance of the actuators themselves is around a $1 \mathrm{kHz}$.

\section{LAB TESTING}

In this section we summarize the suite of tests we plan to do with the ASM. We will focus here on the tests with the fully integrated ASM in the lab and on the telescope with and without the AO systems. Other contributions in this conference discuss tests characterizing the TNO actuators in a 60 -actuator flat deformable mirror ${ }^{11}$ and plans to test a new lab prototype DM based with 19 of the TNO UH88-style actuators ${ }^{9,11}$ with a $39 \mathrm{~mm}$ pitch and a flat facesheet $3.5 \mathrm{~mm}$ thick. These DMs serve as prototypes for the technology and importantly test the fabrication processes.

The first tests of the UH88 ASM assembly will be done at TNO using a set of six capacitive sensors to test basic functionality and provide a first look at the performance of the assembly. The sensors measure the change in position of the facesheet with respect to the ASM body/structure. In this way they are similar to the capacitive sensors in current ASMs except that we are using the sensors to sense differential motion and not as an absolute position reference. To provide flexibility on what region of the ASM is tested, the ASM body will have a series of machined openings into which we can place one of the sensors.

The capacitive sensors provide good temporal resolution $(\sim 10 \mathrm{kHz})$, sub-nm vertical resolution and sparse but sufficient spatial resolution across the surface. Measurements from the sensors can be combined with our finite-element models of the ASM to provide a good first test of the system. In addition to basic functional tests, we can use these to test the basic "static" performance of the system: actuator stroke, repeatability tests, linearity, hysteresis, and inter-actuator coupling (via models). In addition, in combination with our FEA models and accelerometers, we may be able measure the resonant mode frequencies of the ASM. Importantly, the capacitive sensors provide an independent calibration for later tests. In particular the they ground the measurements of the zonal and modal influence functions albeit at a limited number of points about the mirror.

The full optical tests of the ASM normally require a large complex optical setup. These setups serve two functions. They calibrate the static (and possibly dynamic) response of the ASM and they allow the team to integrate and test the ASM with the rest of the AO components (e.g. wavefront sensor(s) and real-time controller). Each of these are important steps that we need to perform with the UH88 ASM. However, such test setups are complex and expensive ${ }^{13}$. For this project we have decided to split the measurement of the static performance of the system (e.g. the zonal/modal influence functions) and the dynamic/AO system integration tests into two separate test setups.

The ASM influence functions (both zonal and modal) require high-spatial resolution measurements that preferably cover the entire surface in each measurement. Both the zonal and modal influence functions are needed in the control of the ASM (e.g. pseudo synthetic interaction matrices and modal control/gains). However, testing the large convex surface of the ASM in one metrology setup presents a challenge. Typical approaches such as a Hindle sphere test or a transmissive variant using a lens ${ }^{13}$ require a large optical element of at least the size of the ASM to test the full surface of the mirror. In our case the Hindle sphere test requires a spherical mirror several meters in diameter. For this project such an optical setup is not possible. Instead to measure the influence functions we'll use an approach called phase measuring deflectometry (PMD). Others have used this for optical metrology ${ }^{14}$ and the principle is commonly used in commercial "structured light" 3D scanners. The basic idea is shown in Figure 2 below. A camera looks at the reflection of a screen off the mirror being tested. The approach effectively traces rays from the screen to the mirror and to the camera. These ray paths depend on the geometry of the setup and the local gradients across the surface of the mirror. We know or can calibrate the geometry of the setup so the gradient of the surface can be derived. These slopes can then be integrated to deduce the surface shape. 
The bookkeeping of the raytracing is done via a judicious choice of the pattern(s) displayed on the screen. A series of two-dimensional sinusoidal patterns with known phase shifts between images in the sequence effectively encode the positions on the screen so they can be tracked to positions on the mirror in the distorted images captured by the camera. This is analogous to the reduction of fringe patterns from interferometers and techniques to unwrap 2-pi ambiguities in the analysis are commonly applied. However, unlike an interferometric fringe pattern, we can display any set of fringes we like (and even different colors) on the screen. Doing so allows us to measure both the $\mathrm{x}$ - and $\mathrm{y}$-slopes with a single pattern. This makes the data acquisition fast though it results in a very high resolution gradient map (e.g. thousands of elements across the mirror). Turning these into a surface shape requires a large matrix multiplication.
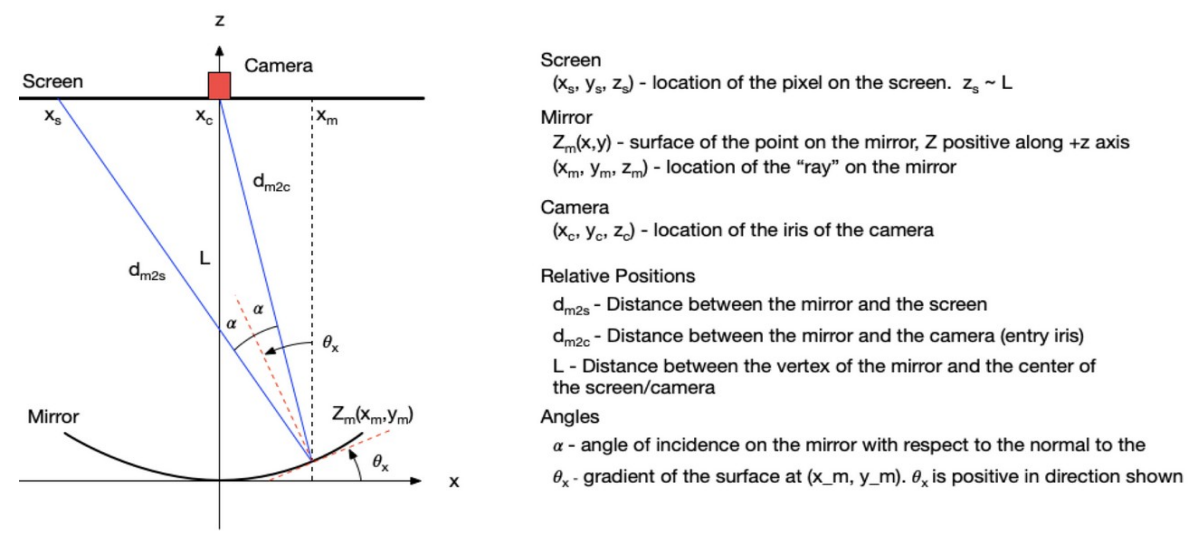

Figure 2. The geometry of the deflectometry setup with the camera positioned along the optical axis of the mirror.

The beauty of the PMD approach is that the required hardware elements are very simple and inexpensive. For the UH88 ASM to cover the entire surface of the mirror in one measurement we need a camera and two $\sim 70$-inch $4 \mathrm{k}$ LCD monitors. This is 2-3 order of magnitude reduction in the cost of the hardware compared to traditional approaches and scales to larger ASMs. The approach was used an LBT $\mathrm{ASM}^{14}$ with results that compare favorably to interferometric approaches.

The challenge with deflectometry is that the position of the camera, mirror, and screens must be known to high precision. Errors in these positions can not be distinguished with low-order aberrations on the surface of the mirror. However, the measurement of the zonal and modal influence functions is inherently differential so as long as the calibration of the setup is stable during the measurements (e.g. poke+ and poke-) then these errors are do not contribute to the estimate of the influence functions.

The deflectometry setup is a lab only test but importantly provides high-resolution full aperture measurements. With it we will repeat many of the basic functionality tests done with the capacitive sensors and also obtain the influence functions. Here the capacitive sensors provide independent point measures of the influence to which we can compare the deflectometry measurements.

Note that we can not perform any measurements of the ASM resonant modes as these are at too a high a temporal frequency for the deflectometry measurements. To do these (and to integrate the ASM with the AO components) we need a second setup. For these tests we will deploy a transmissive lens variant of the Hindle sphere test similar to that done by Stalcup et al for the MMT ASM ${ }^{13}$. For budget reasons we only make these measurements over a portion of the mirror at one time. Figure 3 above shows the approach. A meniscus lens (MHS) smaller than the diameter of the ASM is placed in front of the ASM and acts to divert the beam from an illuminator, placed at the focus of the ASM's hyperboloid, onto the ASM. The back surface the meniscus lens acts as the Hindle sphere and reflects rays bouncing off the ASM back to the front focus. At this location we place our wavefront sensor. With this setup we can not test all actuators at once but we can close the loop on a portion of the mirror in the lab. The MHS lens can be moved to other locations to test other actuators/portions of the ASM. The setup has the additional advantage that it can be used in the lab and when the ASM is installed on the telescope. The illuminator and wavefront sensor will be mounted at the telescope focus and the MHS lens can be installed when the ASM is on the telescope. 


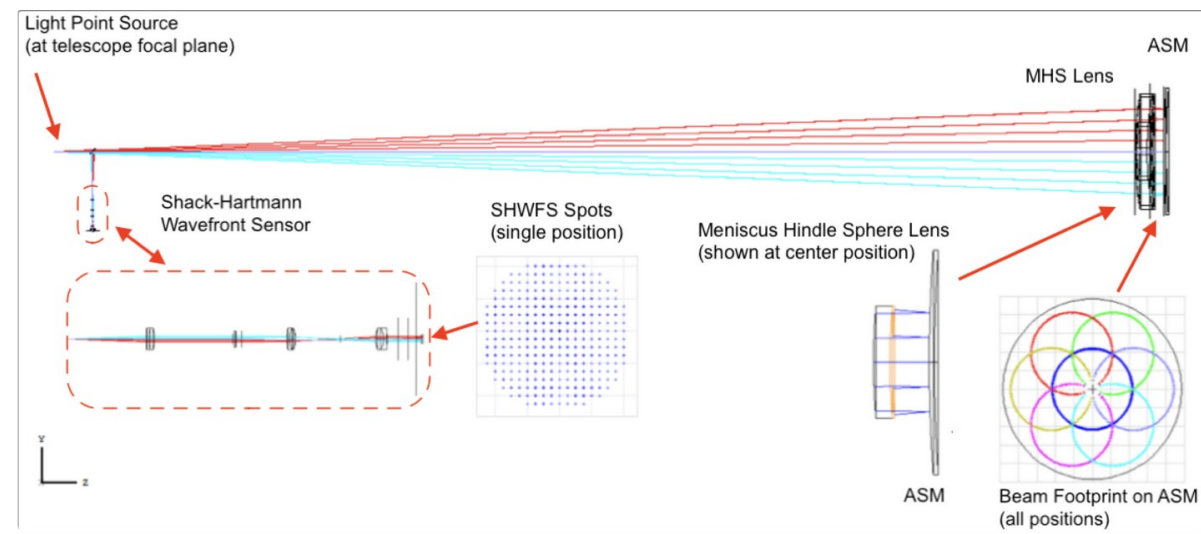

Figure 3. A transmissive Hindle-sphere test setup for the UH88 ASM.

\section{ON-SKY TESTING}

The rapid reconfiguring of the UH 2.2-meter telescope to put the ASM into the optical beam and the fact that we can obtain significant time on sky to test the ASM allows us to step thru the on-sky tests in a simple phased approach.
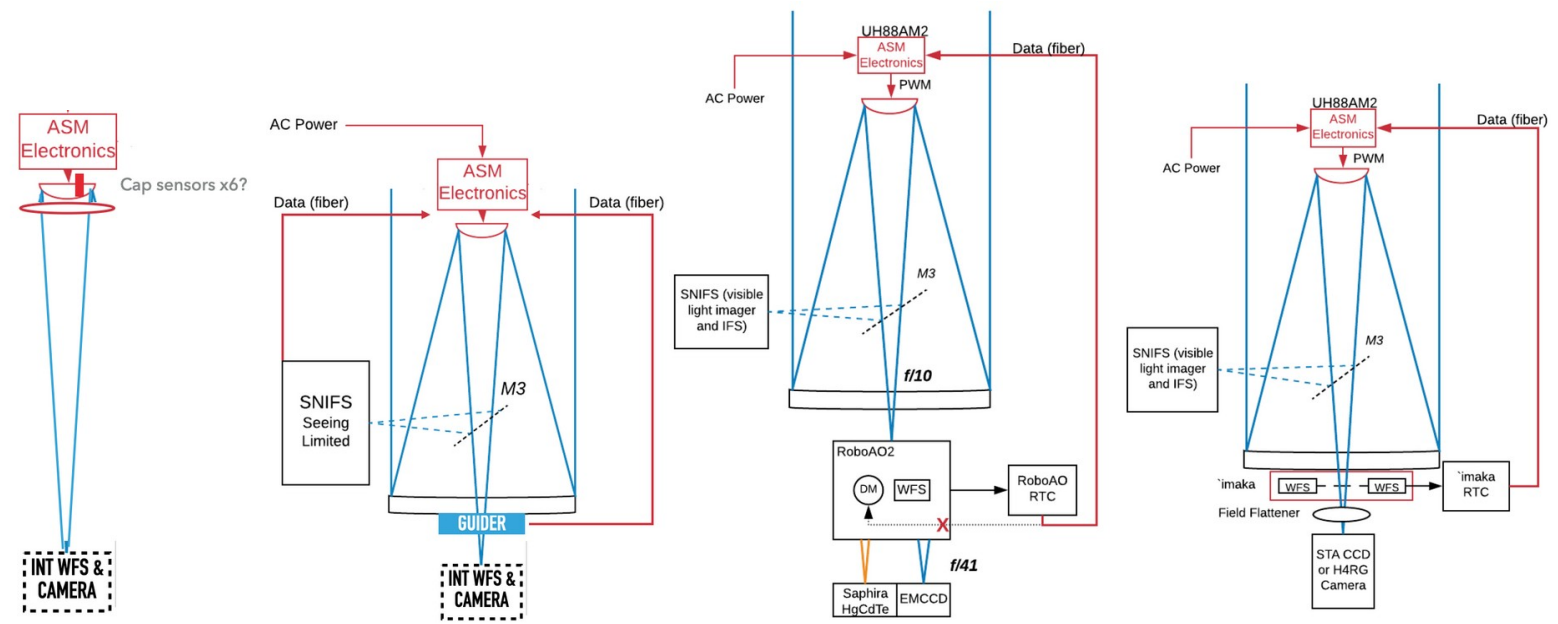

Figure 4. Various configurations of the UH88 ASM from Left to Right: Phase 1 - with the integration WFS and camera, Phase 2 - in seeing-limited mode, Phase 3 - with Robo-AO-2 in SCAO mode with NGS and LGS, and Phase 4 - with imaka in GLAO mode.

In the first phase we test the functionality of the system during the day using the MHS lens setup and then transition to SCAO on a bright natural guide star. The instrument setup is shown on the left in Figure 4. For the daytime tests the Hindle sphere test lens is put in place and at the focus of the telescope we mount the integration WFS, a small-pixel CMOS camera, and the illuminator. This allows us to do simple environmental tests with the ASM (gravity, temperature). We'll also go to the sky for initial tests with this same basic setup, without the Hindle sphere lens to do SCAO on a bright natural guide star. We will start the on-sky tests with this setup since it does not require integration with the RoboAO or 'imaka systems. However, the imaka RTC will be used for the real-time control of the ASM.

The integration WFS and camera also allow us to do seeing limited tests of the ASM. In particular we will quantify how the image quality of the telescope is maintained via a look up table or a simple low-order wavefront sensor (for example a modified telescope guider) that drives the ASM. These tests will mostly be done with the integration wavefront sensor/ 
camera but we can also feed the optical beam to one of our seeing limited instruments to measure image quality over a wider field of view (albeit with less precision on the image quality).

More complete SCAO tests begins when we integrate the ASM with the Robo-AO-2 system. The ASM is sized to be compatible with the Robo-AO-2 natural guide star and laser guide star wavefront sensors and we will modify the control system to send the wavefront correction to either its internal DM or the ASM. This allows us to do a side-by-side comparison of the AO correction by the ASM and by the DM in Robo-AO-2 at visible and near-infrared wavelengths. This will be the first test of the system at longer wavelengths - but note the configuration does not test the thermal emissivity gains of the ASM since RoboAO's optical relay is warm.

Finally, we will use the ASM in a wide-field GLAO configuration. The simplest implementation is to modify the existing 'imaka GLAO system to drive the ASM but still use its 'imaka optical relay. This provides an up to $0.3 \times 0.4 \mathrm{deg}$ field of view for the five NGS WFSs. However, for these tests we plan to remove the imaka optical relay and place the imaka WFSs and science camera at the $\mathrm{f} / 10$ telescope focus. This gives a 0.5 -deg (unvignetted) FOV for the WFS GSs and a science camera that subtends a $0.25 \times 0.25$ degrees.

\section{CONCLUSIONS}

In the coming year we will complete the fabrication of the UH88 ASM and begin testing of the system in Hawaii. We have planned a long road of activities with the system from classical single-conjugate narrow field AO observations in the visible thru near infrared wavelengths, to half-degree field of view ground-layer adaptive optics, and for seeing limited non-adaptive optics observations.

\section{ACKNOWLEDGMENTS}

The UH88 ASM work is funded by the National Science Foundation (AST-1910552).

\section{REFERENCES}

[1] Lloyd-Hart, Brusa, Wildi, et al., "Lessons learned from the first adaptive secondary mirror," Proc. SPIE 5169, Astronomical Adaptive Optics Systems and Applications, (24 December 2003); doi: 10.1117/12.507316, (2003)

[2] Christou, J. et al., "Living with adaptive secondary mirrors 365/7/24," Proc. SPIE 9148, Adaptive Optics Systems IV, 91480F (4 August 2014); doi: 10.1117/12.2056361

[3] Close, L. et al., "Status of MagAO and review of astronomical science with visible light adaptive optics", Proc. of SPIE, 10703 (2018).

[4] Madec et al., "Adaptive Optics Facility: from an amazing present to a brilliant future...", Proc. Of the SPIE, 10703, (2018).

[5] Kuiper, S., Doelman, N., Human, J., Saathof, R., Klop, W., \& Maniscalco, M. (2018, July). Advances of TNO's electromagnetic deformable mirror development. In Advances in Optical and Mechanical Technologies for Telescopes and Instrumentation III Optics and Photonics.

[6] Baranec et al, "High-efficiency Autonomous Laser Adaptive Optics", The Astrophysical Journal Letters, 790(1), L8 (2014).

[7] Baranec et al, "The Robo-AO-2 facility for rapid visible/near-infrared AO imaging and the demonstration of hybrid techniques", Proc. of the SPIE, 10703 (2018).

[8] Chun et al., "On-sky results from the wide-field ground-layer adaptive optics demonstrator 'imaka," Proc. SPIE 10703, Adaptive Optics Systems VI, 107030J (10 July 2018); 
[9] Wouter A. Jonker et al. "Design and manufacturing status of the UH-88 adaptive secondary mirror", Proc SPIE 11451 (2020).

[10] Kuiper S. et al., "Performance Analysis of an adaptive secondary mirror for the University of Hawaii 2.2-meter Telescope", Proc. SPIE 11448, (2020).

[11] Bowens-Rubin. R. et al., "Performance of Large Format-Deformable Mirror Constructed with TNO VariableReluctance Actuators", Proc. SPIE 11448-234, (2020).

[12] Hinz, P. et al., "Developing adaptive secondary mirror concepts for the Shane and Keck Telescopes", Proc. SPIE, 11448-233, (2020).

[13] Stalcup et al., "A test stand for the MMT Observatory adaptive secondary," Proc. SPIE 7015, Adaptive Optics Systems, 701565 (15 July 2008); doi: 10.1117/12.790125

[14] Kolb, Madec, Arsenault et al, "Laboratory results from the AOF system testing", Proc of the SPIE, 9909, (2016).

[15] Huang et al., "Measurement of a large deformable aspherical mirror using SCOTS (Software Configurable Optical Test System)," Proc. SPIE 8838, Optical Manufacturing and Testing X, 883807 (7 September 2013); doi: $10.1117 / 12.2024336$

[16] Kolb, "Review of AO calibrations, or how to best educate your AO system," Proc. SPIE 9909, Adaptive Optics Systems V, 99090K (26 July 2016); doi: 10.1117/12.2232874

[17] Lai et al, "Dynamic On-sky Covariance Random Interaction Matrix Evaluation, a novel method for calibrating adaptive optics systems.", Monthly Notices of the Royal Astronomical Society, submitted (2020) 